\title{
A review of current treatment strategies for restless legs syndrome (Willis-Ekbom disease)
}

\author{
Authors: Lisa Klingelhoefer, ${ }^{\mathrm{A}}$ Ilaria Cova, ${ }^{\mathrm{B}}$ Sheena Gupta ${ }^{\mathrm{C}}$ and Kallol Ray Chaudhuri ${ }^{\mathrm{D}}$
}

\begin{abstract}
Restless legs syndrome (RLS), recently renamed Willis-Ekbom disease (WED), is a common movement disorder. It is characterised by the need to move mainly the legs due to uncomfortable, sometimes painful sensations in the legs, which have a diurnal variation and a release with movement. Management is complex. First, centres should establish the severity of RLS using a simple 10-item RLS severity rating scale (IRLS). They should also exclude secondary causes, in particular ensuring normal iron levels. Mild cases can be managed by lifestyle changes, but patients with a IRLS score above 15 usually require pharmacological treatment. Dopaminergic therapies remain the mainstay of medical therapies, with recent evidence suggesting opioids may be particularly effective. This article focuses on the different treatment strategies in RLS, their associated complications and ways to manage them.
\end{abstract}

KEYWORDS: Restless legs syndrome, RLS, therapy, medical treatment, side effects

\section{Introduction}

Restless legs syndrome (RLS), also known as Willis-Ekbom disease (WED), is a common neurological disorder characterised by an uncontrollable urge to move certain parts of the body, particularly the lower limbs. This is usually accompanied or caused by unpleasant sensations in the legs. ${ }^{1}$ Symptoms of RLS appear or worsen during periods of inactivity or rest and improve with activity. The estimated prevalence of RLS ranges between $5 \%$ and $15 \%$, but figures vary widely depending

\footnotetext{
Authors: Afellow, National Parkinson Foundation International Centre of Excellence, King's College Hospital and King's College, London, UK and resident, Department of Neurology, Technical University Dresden, Dresden, Germany; ${ }^{\mathrm{B}}$ clinical observer and fellow, National Parkinson Foundation International Centre of Excellence, King's College Hospital and King's College, London, UK; research student, National Parkinson Foundation International Centre of Excellence, King's College Hospital and King's College, London, UK; Dprofessor of movement disorders and lead, National Parkinson Foundation International Centre of Excellence, King's College Hospital and King's College, London, UK
}

upon the population surveyed and the severity of symptoms required for inclusion. ${ }^{2,3}$ RLS can be categorised as primary (idiopathic) or secondary. Secondary RLS occurs as a result of certain conditions, such as iron deficiency, pregnancy, end-stage renal disease, diabetes, rheumatoid arthritis and, occasionally, peripheral neuropathies.

\section{Diagnosis}

The diagnosis of RLS is based primarily on the patient's clinical history and on a neurological examination to exclude peripheral neuropathy. The International RLS Study Group (IRLSSG) essential diagnostic criteria for RLS were revised in 2012 and can be used in clinical practice. They include: ${ }^{4}$

1 an urge to move the legs usually accompanied or caused by uncomfortable sensations in the legs

2 worsening of symptoms during times of rest or inactivity

3 partial or total relief of symptoms by movement

4 the symptoms only occur or are worse in the evening or night

5 the occurrence of the above features are not solely accounted for as symptoms primary to another medical or behavioural condition (such as myalgia, venous stasis, leg oedema, arthritis, leg cramps, positional discomfort or habitual foot tapping).

More recently, a single standard question for rapid screening for RLS has been validated. ${ }^{5}$ The question, developed by the IRLSSG on the basis of standard diagnostic criteria, is: 'When you try to relax in the evening or sleep at night, do you ever have unpleasant, restless feelings in your legs that can be relieved by walking or movement?'. As this single question had $100 \%$ sensitivity and $96.8 \%$ specificity for the diagnosis of RLS, it can be used to screen large patient groups effectively. Nevertheless, the final diagnosis should always be confirmed by matching the patient's history and symptoms with the IRLSSG diagnostic criteria, accompanied by the exclusion of secondary conditions.

\section{Treatment}

The IRLSSG rating scale (IRLS) for determining the severity of RLS consists of 10 questions, each being scored into one of five severity categories (from 0 to 4 ), with the maximum total score being $40 .{ }^{6}$ The severity of RLS symptoms is scored as mild (1-10), moderate (11-20), severe (21-30) and very severe (31-40). The patient's score can be used to decide whether pharmacological treatment would be beneficial. 


\section{Non-pharmacological treatment}

The majority of RLS cases are mild and can be managed by lifestyle changes or non-pharmacological treatment. Lifestyle factors known to precipitate RLS symptoms are high caffeine intake ${ }^{7}$ (avoid any intake of caffeine before bedtime), excessive alcohol consumption $^{8}$ (avoid any intake of alcohol before bedtime), severe stress, shift work and intense physical activity near bedtime. Furthermore, sleep hygiene is important and may help patients with RLS. It is important, therefore, for patients with RLS to consider the following as part of their bedtime routine:

> a quiet, comfortable and cool sleeping environment

> appropriate nightwear (such as silk pyjama or dressing gown)

$>$ regular sleep pattern (go to bed and wake up at regular hours, avoid daytime naps)

$>$ associate bed with sleep (for instance, avoid watching TV from bed).

As RLS usually affects the first part of the sleep cycle at night, patients may be advised to alter their sleep pattern by going to bed late and waking up late.

During an attack, patients may benefit from:

$>$ a hot or cold massage, rubbing or massaging the affected limbs ${ }^{9}$

$>$ bathing in hot or cold water

$>$ physical activities such as walking, stretching and exercise

$>$ relaxation exercises (eg biofeedback or yoga)

> distracting the mind by mental exercises (for example, reading an interesting book during the onset of symptoms).

Many other unproven therapies have been described on the basis of individual experience, such as the use of capsaicin cream or magnesium and halcyon bracelets, but these cannot be recommended at this time.

\section{Treating secondary causes}

It is important to distinguish between primary and secondary RLS. Primary RLS tends to run a chronic course and symptoms worsen with time, especially in patients with early onset. By contrast, in secondary RLS symptoms may remit once the secondary cause (eg iron deficiency or end-stage renal disease) is treated. Iron deficiency is an important factor even in primary RLS; therefore, iron parameters (specifically serum ferritin levels) should be measured. Those with low or borderline levels require oral iron treatment for 1 to 2 months with a repeat measurement of serum ferritin levels to avoid iron overload. ${ }^{10}$ Box 1 summarises blood tests necessary to exclude secondary causes of RLS.

Patients with severe RLS and insomnia may require sleep studies such as polysomnography ${ }^{11}$ or immobilisation tests, ${ }^{12,13}$ but in practice these are rarely performed in the UK.

\section{Avoiding medication that exacerbate restless legs syndrome}

Several drugs are known to worsen RLS symptoms and, if possible, these should be avoided. Medications that are known to induce or exacerbate RLS symptoms include: antihistamines, dopamine antagonists, anti-nausea medications, anxiolytics, antidepressants (such as mirtazapine, escitalopram, fluoxetine and mianserin), neuroleptics (such as olanzapine), beta-blockers,
Box 1. Recommended blood tests to rule out

secondary restless legs syndrome.

Iron studies (specifically serum ferritin level is essential)

Full blood count (to exclude anaemia)

Serum vitamin $B_{12}$ and folic acid

Serum glucose and $\mathrm{HbA} 1 \mathrm{C}$

Urea and electrolytes

Serum creatinine

Albumin levels (optional)

Thyroid function tests (optional)

anticonvulsants, L-thyroxine and lithium. ${ }^{14}$ In clinical practice, it is useful to enquire about medication intake and taper down the doses of any antihistamines or dopamine antagonists that are being used.

\section{Pharmacological treatment}

An estimated $10-15 \%$ of RLS patients have symptoms severe enough to require medical management of RLS. ${ }^{15}$ Treatment is only required when the symptoms are clinically significant. All possible treatment options only address the symptoms and are not preventive. Furthermore, as RLS is a chronic disorder, it is likely that once the patient starts medication, they will continue to use it for the rest of their lives. Therefore, RLS should be treated in order to alleviate sensory and motor symptoms, to improve sleep and quality of life and to prevent cardiovascular complications. The dosage should be kept as low as possible.

Currently, dopaminergic agents are used as first-line treatment for moderate to severe RLS. Randomised placebo controlled studies have established the efficacy of dopamine agonists (DA) in RLS for both improvement of symptoms and reduction in periodic leg movements during sleep and awakening. ${ }^{16}$ The European Federation of Neurological Societies has recommended non-ergot DAs, such as ropinirole and pramipexole as tablets or rotigotine as patches. In the UK, DAs are the only licensed treatment for RLS. The different DAs and their recommended doses are summarised in Table 1. The doses are considerably smaller than those used for patients with Parkinson's disease. DAs should be administered as a single evening dose and are effective for the short- and long-term treatment of idiopathic RLS. If the patient shows intolerance to one agent, then another DA should be tried. Oral therapies such as pramipexole and ropinirole are both effective, but their use depends on tolerability and efficacy. Rotigotine patches have the advantage of being efficacious during both the day and night, and many clinicians prescribe the patch once oral therapies have failed. DAs alleviate symptoms in up to $70 \%$ of patients.

Levodopa is used occasionally as the first-line treatment for intermittent RLS symptoms (less than three times a week) in Germany, Austria and Switzerland. It is mainly used as a rescue medication and so is taken when symptoms occur or when they are anticipated. Levodopa therapy is associated with a high rate of side effects, such as augmentation (an overall increase in RLS symptom severity) (60-80\%), ${ }^{17}$ and is not recommended as first-line treatment in the UK. Levodopa can also be used as a clinical 'challenge test' for the diagnosis of RLS. ${ }^{18}$ 


\begin{tabular}{|c|c|c|c|c|}
\hline Medication & $\begin{array}{l}\text { Minimal starting } \\
\text { dose/maximal } \\
\text { recommended dose }\end{array}$ & $\begin{array}{l}\text { Time to full } \\
\text { effect of the } \\
\text { therapeutic dose }\end{array}$ & Half-life & Side effects \\
\hline Levodopa & $50 \mathrm{mg} / 200 \mathrm{mg}$ & At first dose & $1.5-2$ hours & $\begin{array}{l}\text { High rates of augmentation and loss of efficacy with rebound } \\
\text { phenomena. }\end{array}$ \\
\hline Ropinirole & $0.25 \mathrm{mg} / 4 \mathrm{mg}$ & $4-10$ days & 6 hours & $\begin{array}{l}\text { Augmentation, impulse control disorder, nausea, low blood pressure, } \\
\text { dizziness, headache, nasal congestion, sleepiness in susceptible patients. }\end{array}$ \\
\hline Pramipexole & $0.125 \mathrm{mg} / 0.54 \mathrm{mg}$ & At first dose & $8-12$ hours & $\begin{array}{l}\text { Augmentation, impulse control disorder, nausea, low blood } \\
\text { pressure, dizziness, headache, nasal congestion, sleepiness in } \\
\text { susceptible patients. }\end{array}$ \\
\hline Rotigotine & $1 \mathrm{mg} / 3 \mathrm{mg}$ & 1 week & $5-7$ hours & $\begin{array}{l}\text { Skin irritation, low risk of augmentation, nausea, low blood } \\
\text { pressure, dizziness, headache, nasal congestion, sleepiness in } \\
\text { susceptible patients. Risk of ICD may be lower. }\end{array}$ \\
\hline Pregabalin & $25 \mathrm{mg} / 300 \mathrm{mg}$ & $3-6$ days & 10 hours & Sleepiness, dizziness, headache, fluid retention. \\
\hline Clonazepam & $0.50 \mathrm{mg} / 2.0 \mathrm{mg}$ & $\begin{array}{l}\text { First dose: effect } \\
\text { mainly on sleep }\end{array}$ & $30-40$ hours & High risk of sleepiness, dizziness, morning drug hangover. \\
\hline Gabapentin & $300 \mathrm{mg} / 2,700 \mathrm{mg}$ & $3-6$ days & $5-7$ hours & Sleepiness, dizziness, fluid retention. \\
\hline Tramadol & $25 \mathrm{mg} / 100 \mathrm{mg}$ & N/A & $5-8$ hours & Dizziness, sleepiness, constipation, dry mouth \\
\hline Oxycodone & $2.5 \mathrm{mg} / 25 \mathrm{mg}$ & N/A & $2-4$ hours & Dizziness, fatigue, constipation, nausea \\
\hline
\end{tabular}

Second-line off-label treatment options for daily RLS symptoms are $\alpha 2 \delta$ ligands (such as gabapentin or pregabalin) and opioids (such as oxycodone, propoxyphene, tramadol, tilidine or codeine). These are particularly useful for patients with painful RLS symptoms, coexisting neuropathy or pain elsewhere. ${ }^{19} \mathrm{~A}$ recent study found that prolonged-release oxycodone-naloxone caused an 8-point reduction in the mean IRLS sum score compared to placebo. This represents one of the most effective treatment responses ever seen in RLS, and so the use of this combined medication in severe RLS is likely to be adopted. ${ }^{20}$

Table 1 summarises the different medications used for the treatment of RLS, their recommended minimum start dose, maximum dose and main important side effects.

\section{Follow up}

RLS patients should be followed up regularly to monitor treatment efficacy, as the disease tends to worsen over time. The European Restless Legs Study Group (EURLSSG) recommends that even in the absence of complications, patients need to see their physician every 6-12 months. A symptom diary completed over 7-14 days prior to the consultation could provide the physician with details about the severity of symptoms and the effect of treatment.

If a patient complains of worsening of RLS symptoms while being on treatment, the physician should check whether the patient is compliant, if they are taking any additional medication that may exacerbate symptoms or whether they have become more sedentary as this can cause symptoms to appear earlier during the day. It is important to check iron parameters during follow-up consultations as iron deficiency is implicated in the onset as well as the severity of RLS. ${ }^{10}$

\section{Treatment complications}

\section{Early morning rebound}

Early morning rebound is the reappearance of RLS symptoms in the early morning as the effects of medication are wearing off. Symptoms tend to be worse than expected if no medication was taken but they disappear several hours later. Early morning rebound occurs more frequently with short half-life medication such as levodopa. ${ }^{21}$ It is important to exclude concurrent conditions, such as depression or sleep apnoea, before modifying the current treatment. To manage this complication, the dose of the currently prescribed drug can be increased, the time of administration can be changed or another dopaminergic agent with a longer half-life can be prescribed. ${ }^{22}$

\section{Tolerance and loss of efficacy}

Tolerance consists of a decrease in the response to a drug over time, requiring an increase in dose to achieve the original amelioration of symptoms. This is to be differentiated from the natural worsening of RLS symptoms, which would also require higher drug doses. Unlike rebound or augmentation, symptoms are not worse than before treatment initiation. It is not completely known whether tolerance inevitably precedes and leads to augmentation.

\section{Augmentation}

Augmentation is a treatment-related side effect that is characterised by an overall increase in RLS symptom severity. It is the worst long-term complication of dopaminergic treatment 
Table 2. Max Planck Institute (MPI) diagnostic criteria for augmentation. Adapted with permission from Garcia-Borreguero et al (2007).23

\author{
Augmentation requires criteria $\mathrm{A}+\mathrm{B}$ or $\mathrm{A}+\mathrm{C}$ or $\mathrm{A}+\mathrm{B}+\mathrm{C}$ to be met \\ A) Basic criteria \\ $>$ Increase in symptom severity experienced on 5 out of 7 days during the previous week. \\ > Increase in symptom severity is not accounted by any other factors (such as change in medication, lifestyle or natural progression of RLS). \\ $>$ Positive response to prior treatment.
}

\title{
In addition, either $\mathrm{B}$ or $\mathrm{C}$ or both have to be met.
}

B) Persisting paradoxical response to treatment: RLS symptom severity increases some time after a dose increase and improves some time after a dose decrease.

C) Earlier onset of symptoms

$>$ by at least 4 hours

> between 2 and 4 hours plus one of the following points i-iv compared to symptom status before treatment:

i shorter latency to symptoms when at rest

ii extension of symptoms to other body parts

iii greater intensity of symptoms (or increase in periodic limb movements could be measured by polysomnography or immobilisation test)

iv shorter duration of relief from treatment.

of RLS and leads to worse symptoms than were present before treatment had been started. The Max Planck Institute (MPI) diagnostic criteria for augmentation are listed in Table 2.

Augmentation occurs more frequently in patients who take daily doses higher than the following: $200 \mathrm{mg}$ levodopa, $0.5 \mathrm{mg}$ pramipexole, $2 \mathrm{mg}$ ropinirole or $3 \mathrm{mg}$ rotigotine. The rate of augmentation is greatest with levodopa and among the DAs, it is greatest with pramipexole and lowest with rotigotine with an incidence of approximately $2-3 \%$ per year. ${ }^{24}$ Although the vast majority of augmentation cases are due to dopaminergic agents, a few cases have been reported with the use of tramadol. ${ }^{14,24}$ No other medication has been found to cause augmentation. Predictors of augmentation have been identified as low plasma ferritin levels, previous episodes of augmentation, longer treatment duration and possibly a preceding tolerance to medication. ${ }^{24,25}$

Several approaches can be taken to prevent augmentation. The most effective preventative measure is to prescribe the lowest effective dose of dopaminergic medication. Ideally, a low dose of a long-acting dopaminergic agent (such as rotigotine) should be used; conversely, agents with high rates of augmentation (such as levodopa) should be avoided. Another measure is to divide the dopaminergic dose, administering the first dose earlier in the afternoon before symptom onset and the second dose after symptoms started. Divided dose of RLS medication has not, however, been approved yet. As low iron body stores may be responsible for the augmentation, iron parameters should be measured and an oral iron intake must be considered if serum ferritin is below $50 \mu \mathrm{g} / \mathrm{l}$.

When the measures described above are unsuccessful and symptoms are so severe that they interfere with the patient's daily activities, a different DA may be prescribed. Augmentation is, however, likely to reoccur even when another dopaminergic agent is used. If changing dopaminergic agents does not improve symptoms, the dopaminergic dose should be further reduced while simultaneously initiating treatment with a non-dopaminergic agent (such as an $\alpha 2 \delta$ ligand or an opiate), until the dopaminergic agent is discontinued altogether. ${ }^{26}$

\section{Impulse control disorders}

Impulse control disorders (ICD) are a well-known side effect of dopaminergic agents in patients with Parkinson's disease. ${ }^{27}$ They have also recently been described in RLS, despite the lower doses of dopaminergic medication used by these patients. ICDs during RLS treatment are estimated to affect between 3\% and $17 \%$ of patients and include obsessive-compulsive behaviour, hypersexuality, binge eating, pathologic gambling, punding and compulsive shopping. ${ }^{28-30}$ The incidence of ICDs is dosedependent and ceases when dopaminergic treatment is stopped. If ICDs occur during treatment with a dopaminergic agent, the medication should be either discontinued or progressively decreased until the side effect resolves, or until another nondopaminergic drug can be substituted or added.

\section{Weight gain}

Sleep loss has been shown to be related to weight gain and obesity. ${ }^{31}$ Between $20 \%$ and $30 \%$ of patients with RLS eat during the night. It is important to differentiate weight gain, fluid retention (oedema) and binge eating. Weight gain is also a common side effect of $\alpha 2 \delta$ ligands and appears to be dose-dependent. Water retention can also occur with dopaminergic agents.

\section{Mood changes}

Depression and anxiety are common comorbid conditions associated with RLS. ${ }^{32}$ Patients with RLS, especially those who are taking $\alpha 2 \delta$ ligands, are at greater risk of developing depression than healthy individuals. Furthermore, augmentation is a common trigger of anxiety. If depression 
occurs, the physician should avoid prescribing an antidepressant that is known to worsen RLS symptoms, such as a selective norepinephrine reuptake inhibitor (SNRI) or a selective serotonin reuptake inhibitor (SSRI). Suitable antidepressants include duloxetine, buproprion, lamictal, trazadone and desipramine. Cognitive behaviour therapy can also be of benefit.

\section{Conclusions}

RLS is a common condition and can have a great impact on quality of life, mainly because of disturbed night time sleep and daytime sleepiness. Treatment depends on the severity and frequency of symptoms, which can be determined by using the IRLSSG rating scale. Mild RLS may be managed with reassurance and lifestyle changes. Moderate to severe cases require pharmacological treatment. Secondary causes and exacerbating factors should be identified and treated. Patients with poorly managed RLS can suffer from severe morbidity and a poor quality of life associated with marked anxiety and, sometimes, depression.

\section{Conflict of interest}

Lisa Klingelhoefer, Ilaria Cova and Sheena Gupta declare that there are no competing interests concerning this article. Kallol Ray Chaudhuri is a founding member of RLS UK and declares that there are no competing interests concerning this article.

\section{References}

1 Allen RP, Picchietti D, Hening WA et al. Restless legs syndrome: diagnostic criteria, special considerations, and epidemiology. A report from the restless legs syndrome diagnosis and epidemiology workshop at the National Institutes of Health. Sleep Med 2003;4:101-19.

2 Nichols DA, Allen RP, Grauke JH et al. Restless legs syndrome symptoms in primary care: a prevalence study. Arch Intern Med 2003;163:2323-9.

3 Allen RP, Bharmal M, Calloway M. Prevalence and disease burden of primary restless legs syndrome: results of a general population survey in the United States. Mov Disord 2011;26:114-20.

4 International Restless Legs Syndrome Study Group. 2012 Revised IRLSSG Diagnostic Criteria for RLS. http://irlssg.org/diagnosticcriteria/ [Accessed 31 July 2014].

5 Ferri R, Lanuzza B, Cosentino FI et al. A single question for the rapid screening of restless legs syndrome in the neurological clinical practice. Eur J Neurol 2007;14:1016-21.

6 Walters AS, LeBrocq C, Dhar A et al. Validation of the International Restless Legs Syndrome Study Group rating scale for restless legs syndrome. Sleep Med 2003;4:121-32.

7 Lutz EG. Restless legs, anxiety and caffeinism. J Clin Psychiatry 1978;39:693-8.

8 Aldrich MS, Shipley JE. Alcohol use and periodic limb movements of sleep. Alcohol Clin Exp Res 1993;17:192-6.

9 Russell M. Massage therapy and restless legs syndrome. J Bodywork Mov Ther 2007;11:146-50.

10 Sun ER, Chen CA, Ho G, et al. Iron and the restless legs syndrome. Sleep 1998;21:371-7.

11 Michaud M, Paquet J, Lavigne G et al. Sleep laboratory diagnosis of restless legs syndrome. Eur Neurol 2002;48:108-13.

12 Montplaisir J, Boucher S, Nicolas A et al. Immobilization tests and periodic leg movements in sleep for the diagnosis of restless leg syndrome. Mov Disord 1998;13:324-9.

13 Garcia-Borreguero D, Kohnen R, Boothby L et al. Validation of the Multiple Suggested Immobilization Test: a test for the assessment of severity of restless legs syndrome (Willis-Ekbom Disease). Sleep 2013;36:1101-9.
14 Hoque R, Chesson AL Jr. Pharmacologically induced/exacerbated restless legs syndrome, periodic limb movements of sleep, and REM behavior disorder/REM sleep without atonia: literature review, qualitative scoring, and comparative analysis. J Clin Sleep Med 2010;6:79-83.

15 Hening W, Walters AS, Allen RP et al. Impact, diagnosis and treatment of restless legs syndrome (RLS) in a primary care population: the REST (RLS epidemiology, symptoms, and treatment) primary care study. Sleep Med 2004;5:237-46.

16 Garcia-Borreguero D, Kohnen R, Silber MH et al. The long-term treatment of restless legs syndrome/Willis-Ekbom disease: evidencebased guidelines and clinical consensus best practice guidance: a report from the International Restless Legs Syndrome Study Group. Sleep Med 2013;14:675-84.

17 Högl B, Garcia-Borreguero D, Kohnen R et al. Progressive development of augmentation during long-term treatment with levodopa in restless legs syndrome: results of a prospective multi-center study. J Neurol 2010;257:230-7.

18 Stiasny-Kolster K, Kohnen R, Moller JC et al. Validation of the 'L-DOPA test' for diagnosis of restless legs syndrome. Mov Disord 2006;21:1333-9.

19 Garcia-Borreguero D, Stillman P, Benes $\mathrm{H}$ et al. Algorithms for the diagnosis and treatment of restless legs syndrome in primary care. BMC Neurol 2011;11:28.

20 Trenkwalder $\mathrm{C}$, Benes $\mathrm{H}$, Grote L et al. Prolonged release oxycodonenaloxone for treatment of severe restless legs syndrome after failure of previous treatment: a double-blind, randomised, placebo-controlled trial with an open-label extension. Lancet Neurol 2013;12:1141-50.

21 Guilleminault C, Cetel M, Philip P. Dopaminergic treatment of restless legs and rebound phenomenon. Neurology 1993;43:445.

22 Garcia-Borreguero D, Odin P, Schwarz C. Restless legs syndrome: an overview of the current understanding and management. Acta Neurol Scand 2004;109:303-17.

23 Garcia-Borreguero D, Allen RP, Kohnen R et al. Diagnostic standards for dopaminergic augmentation of restless legs syndrome: report from a World Association of Sleep Medicine-International Restless Legs Syndrome Study Group consensus conference at the Max Planck Institute. Sleep Med 2007;8:520-30.

24 Garcia-Borreguero D, Williams AM. Dopaminergic augmentation of restless legs syndrome. Sleep Med Rev 2010;14:339-46.

25 Trenkwalder C, Hogl B, Benes H, Kohnen R. Augmentation in restless legs syndrome is associated with low ferritin. Sleep Med 2008;9:572-4.

26 Garcia-Borreguero D, Allen RP, Benes $\mathrm{H}$ et al. Augmentation as a treatment complication of restless legs syndrome: concept and management. Mov Disord 2007;22(Suppl 18):S476-84.

27 Antonini A, Cilia R. Behavioural adverse effects of dopaminergic treatments in Parkinson's disease: incidence, neurobiological basis, management and prevention. Drug Saf 2009;32:475-88.

28 Cornelius JR, Tippmann-Peikert M, Slocumb NL et al. Impulse control disorders with the use of dopaminergic agents in restless legs syndrome: a case-control study. Sleep 2010;33:81-7.

29 Schreglmann SR, Gantenbein AR, Eisele G, Baumann CR. Transdermal rotigotine causes impulse control disorders in patients with restless legs syndrome. Parkinsonism Relat Disord 2012;18:207-9.

30 Lipford MC, Silber MH. Long-term use of pramipexole in the management of restless legs syndrome. Sleep Med 2012;13:1280-5.

31 Chaput JP, Despres JP, Bouchard C, Tremblay A. The association between sleep duration and weight gain in adults: a 6-year prospective study from the Quebec Family Study. Sleep 2008;31:517-23.

32 Winkelmann J, Prager M, Lieb R et al. 'Anxietas tibiarum' Depression and anxiety disorders in patients with restless legs syndrome. J Neurol 2005;252:67-71.

Address for correspondence: Dr L Klingelhoefer, National Parkinson Foundation International Centre of Excellence, Department of Neurology, King's College Hospital and King's College, Denmark Hill, London, UK.

Email: lisa.klingelhoefer@nhs.net 